\title{
REVIEWS
}

Check for updates

\section{Transcriptional regulation of memory B cell differentiation}

\author{
Brian J. Laidlaw $\mathbb{D}^{1 凶}$ and Jason G. Cyster ${ }^{2,3}$
}

Abstract | Memory B cells (MBCs) are critical for the rapid development of protective immunity following re-infection. MBCs capable of neutralizing distinct subclasses of pathogens, such as influenza and HIV, have been identified in humans. However, efforts to develop vaccines that induce broadly protective MBCs to rapidly mutating pathogens have not yet been successful. Better understanding of the signals regulating $\mathrm{MBC}$ development and function are essential to overcome current challenges hindering successful vaccine development. Here, we discuss recent advancements regarding the signals and transcription factors regulating germinal centre-derived MBC development and function.

Germinal centre

A structure that forms in the $B$ cell follicle of secondary lymphoid organs during an immune response in which $B$ cells engage with antigen and compete for signals necessary for their survival, proliferation and differentiation into plasma cells or memory B cells.

Somatic hypermutation A cellular process in which proliferating B cells accumulate mutations in antibody complementarity-determining regions, potentially impacting their ability to recognize antigen.
'Division of Allergy and Immunology, Department of Medicine, Washington University School of Medicine, St. Louis, MO, USA.

${ }^{2}$ Department of Microbiology and Immunology, University of California, San Francisco, San Francisco, CA, USA.

${ }^{3}$ Howard Hughes Medical Institute, University of California, San Francisco, San Francisco, CA, USA.

凶e-mail:brian.laidlaw@ wustl.edu

https://doi.org/10.1038/ s41577-020-00446-2
During an immune response, B cells that encounter their cognate antigen become activated and differentiate to form short-lived antibody-secreting cells or germinal centre (GC)-independent memory B cells (MBCs). Within the GC, B cells engage with antigen and compete for limiting amounts of T cell help, which is necessary for B cell survival, proliferation and eventual differentiation into plasma cells or GC-derived $\mathrm{MBCs}^{1}$. The GC is also the primary site in which B cells undergo somatic hypermutation, with B cells that accrue productive mutations preferentially receiving $\mathrm{T}$ cell help. The GC response is required for the development of affinity-matured plasma cells and MBCs (BOX 1).

MBCs are an important component of protective immunity. MBCs are distinguished by their capacity to survive long term and to rapidly differentiate into antibody-secreting cells upon antigen re-encounter. MBCs can also re-enter the GC during recall responses, where they undergo further somatic hypermutation ${ }^{2-5}$. MBCs tend to emerge from the GC during the early phases of the GC response and typically display reduced levels of somatic hypermutation and affinity maturation relative to plasma cells ${ }^{6-8}$. In the context of viral infections, the reduced mutational load of MBCs allows these cells to maintain enhanced flexibility in their responsiveness to different viral subtypes compared with plasma cells, which tend to be specific for a particular subtype. Indeed, the MBC population contains an elevated fraction of broadly reactive clones relative to the plasma cell pool for numerous pathogens in both mice and humans ${ }^{9-12}$. The MBC response comprises multiple subsets, identified based on their expression of CD80 and PDL2, among other markers ${ }^{5,8}$ (BOX 2). These MBC subsets emerge from the GC at different times and vary in their capacity to re-enter the GC or differentiate into antibody-secreting cells upon antigen re-encounter ${ }^{5,6}$.
Iterative exposure to cross-reactive viral antigens is an emerging vaccination strategy designed to elicit broadly reactive MBCs capable of mediating heterosubtypic immunity against pathogens such as influenza. The potential efficacy of an iterative vaccination strategy is limited by the relative inefficiency with which most $\mathrm{MBC}$ clones re-enter the GC response ${ }^{13-15}$. The secondary GC response tends to consist largely of recently activated naive B cells, with only certain MBC subsets possessing the capacity to efficiently re-enter the $\mathrm{GC}^{4,5,16}$. Currently, it is unclear why the majority of MBCs fail to participate in secondary GC responses. One possibility is that antigen-specific antibodies limit the ability of MBC clones of the same specificity to access antigen and participate in the GC response $\mathrm{e}^{4,17}$.

MBCs can arise through both GC-dependent and GC-independent pathways ${ }^{18-20}$. GC-independent MBCs largely develop during the early stages of the immune response and contribute to protective immunity against numerous pathogens including Ehrlichia muris and malaria ${ }^{14,21}$. GC-independent MBCs can be somatically hypermutated but are not thought to undergo affinity maturation, a process that is largely limited to GC-dependent $\mathrm{MBCs}^{14,18,20}$. GC-independent MBCs are also largely not class-switched ${ }^{14,20}$. By contrast, GC-derived MBCs can undergo affinity maturation and are often class-switched, allowing these cells to have an enhanced ability to mediate clearance of some pathogens. Here, we focus on GC-dependent MBCs.

Considerable progress has been made in recent years in elucidating the interactions and signalling pathways that regulate the GC B cell response. However, a better understanding of the mechanisms that govern MBC development and function is needed for the design of vaccines capable of eliciting broadly reactive MBCs that robustly participate in recall responses. In this Review, 


\section{Box $1 \mid$ Germinal centre response}

Within the germinal centre (GC), B cells compete for antigen and limiting amounts of T cell help (delivered via CD40 ligand and cytokines). Higher-affinity B cells tend to capture more antigen, receive more $T$ cell help and, subsequently, migrate from the light zone, where $T$ cells reside, to the dark zone ${ }^{1}$. Within the dark zone, $B$ cells undergo rapid proliferation and somatic hypermutation, with $B$ cells that accrue productive mutations returning to the light zone for continued selection and eventual differentiation into plasma cells or memory B cells ${ }^{104,105}$. B cells that acquire damaging mutations or that are not selected by $T$ cells undergo apoptosis, leading to a progressive increase in B cell affinity over the course of the GC response ${ }^{104,105}$. The enzyme activation-induced cytidine deaminase (AID) mediates somatic hypermutation and class-switch recombination. AID expression is promoted by the transcription factors basic leucine zipper transcription factor (BATF), PAX5, transcription factor 3 (TCF3) and interferon regulatory factor 8 (IRF8) and is inhibited by the transcriptional inhibitors inhibitor of DNA binding 2 (ID2) and ID3 (REFS $38,88,140,141)$.

\section{Plasma cells \\ Large, terminally differentiated B lymphocytes that continually secrete antibodies and are also known as antibody-secreting cells. \\ Recall responses \\ Immune responses in which memory B cells re-encounter their cognate antigen and differentiate into antibody- secreting cells or re-enter the germinal centre to undergo further diversification.}

\section{Affinity maturation \\ The process by which germinal centre B cells increase their affinity for antigen during an immune response as a result of competition for a limiting amount of CD40L-expressing follicular helper T cells.}

Cross-reactive viral antigens Viral protein sequences that are conserved between different strains of a pathogen.

Heterosubtypic immunity Immunity in which lymphocytes are generated that can protect against multiple subtypes of a pathogen

MBC clones

Memory B cells (MBCs) with a particular specificity for antigen.

Secondary GC response

A new germinal centre

(GC) that forms against an immunogen or pathogen that the host previously encountered and developed immunological memory against

Naive B cells

$B$ cells that have not been exposed to antigen. we discuss the transcriptional regulation of the GC response with a focus on recent studies that provide insight into how GC B cells make the decision to differentiate into MBCs. We start by exploring how GC $\mathrm{B}$ cell commitment, maintenance and differentiation into MBCs are regulated transcriptionally. We then outline potential models of MBC differentiation, concluding with a discussion of important areas of future investigation.

\section{Regulation of GC B cell commitment}

To differentiate into GC B cells, naive B cells need to receive simultaneous signals from the antigen-engaged $\mathrm{B}$ cell receptor (BCR) and from CD40L and cytokineexpressing follicular helper $\mathrm{T}\left(\mathrm{T}_{\mathrm{FH}}\right)$ cells. Receipt of these signals allows $\mathrm{B}$ cells to upregulate the zinc finger transcription factor B cell lymphoma 6 (BCL-6), which is required for GC development ${ }^{22}$ (FIG. 1). BCL-6 functions primarily as a transcriptional repressor that controls B cell positioning by negatively regulating the expression of cell migratory receptors, such as sphingosine-1-phosphate receptor 1 (S1PR1) and Epstein-Barr virus-induced G-protein-coupled receptor 2 (EBI2; also known as GPR183) ${ }^{23}$. BCL-6 also induces the expression of S1PR2, which promotes B cell confinement to the $\mathrm{GC}^{23}$. Through direct and indirect mechanisms, BCL- 6 regulates the expression of a wide network of genes controlling cellular processes including the DNA damage response, apoptosis, BCR and CD40 signalling, plasma cell differentiation and T cell:B cell interactions ${ }^{24-27}$. T cell-derived IL-4 and IL-21 act directly on B cells and signal through signal transducer and activator of transcription 6 (STAT6) and STAT4, respectively, to promote BCL-6 expression ${ }^{28-31}$.

Numerous other transcription factors also promote GC commitment through B cell-intrinsic mechanisms, including interferon regulatory factor 4 (IRF4), IRF8, POU class 2 homeobox associating factor 1 (POU2AF1; also known as OBF1), MYC and myocyte enhancer binding factor $2 \mathrm{~b}$ (MEF2B) and MEF2C. IRF4 is necessary for the initiation, but not the maintenance, of the GC response ${ }^{32-34}$. Transient expression of IRF4 in $\mathrm{B}$ cells induces the expression of BCL- 6 and POU2AF1 and promotes GC development ${ }^{32}$. IRF4 expression is rapidly induced by BCR stimulation ${ }^{35}$. IRF4 expression can also be induced by the co-stimulatory protein CD40, which in turn activates the transcription factor nuclear factor- $\kappa \mathrm{B}(\mathrm{NF}-\kappa \mathrm{B})^{36}$. Sustained IRF4 expression is sufficient to directly repress BCL- 6 expression and promote plasma cell differentiation, which may reflect the dose-dependent function of IRF4 in B cells ${ }^{36,37}$.

IRF8 regulates GC B cell development through the direct induction of BCL- 6 expression and can promote GC B cell survival through the induction of the E3 ubiquitin ligase MDM2 (REFS ${ }^{38-40}$ ). IRF8 forms complexes with transcription factors such as SPI-1 (also known as PU.1), which allow it to efficiently bind to target DNA sequences and regulate gene expression ${ }^{41}$. Whereas ablation of IRF8 alone in B cells is not sufficient to overtly impair GC development, compound loss of both IRF8 and PU.1 results in an almost complete loss of GC development ${ }^{42}$. These data suggest that IRF8/ PU.1-mediated induction of BCL- 6 early after activation is an important regulator of GC initiation.

The co-activator OBF1, which forms a complex with the transcription factors POU class 2 homeobox 1 (POU2F1; also known as OCT1) and POU2F2 (also known as OCT2), plays a central role in GC development as deficiency in OBF1 and/or OCT2 results in an inability to form $\mathrm{GCs}^{43,44}$. OCT2 directly regulates the expression of the tyrosine kinase SYK and indirectly regulates the expression of the signalling molecule SLAMF1, which have important roles in BCR signalling and T cell:B cell interactions, respectively ${ }^{45}$. OBF1 and OCT2 induce $B$ cell expression of IL-6, which can promote $T_{F H}$ cell differentiation ${ }^{46}$. The E26 transformation-specific (ETS) transcription factor SPI-B is a direct target of OBF1 and is necessary for GC initiation ${ }^{47-49}$. SPI-B functions in complex with PU.1 to enable B cells to respond appropriately to environmental cues by regulating the expression of BCR signalling pathway components, the receptor CD40, B cell activating factor receptor (BAFFR) and Toll-like receptors (TLRs) ${ }^{50}$.

MYC is required for both the initiation and the maintenance of the GC response ${ }^{51,52}$. MYC is an important regulator of cell proliferation, with $\mathrm{MYC}^{+} \mathrm{B}$ cells expressing higher levels of cell cycle-promoting genes such as cyclin D2 (Ccnd2) and cyclin D3 (Ccnd3) ${ }^{51}$. MYC also induces the expression of E2F transcription factor 1 $(\mathrm{E} 2 \mathrm{~F} 1)^{53}$. E2F1 promotes GC B cell differentiation through the induction of the histone methyltransferase enhancer of zeste homologue $2(\mathrm{EZH} 2)^{54}$. EZH2 is the enzymatic component of the Polycomb repressive complex 2 (PRC2) and is responsible for histone 3 trimethylated at lysine 27 (H3K27me3). EZH2 promotes GC $\mathrm{B}$ cell proliferation via the repression of genes encoding the cyclin-dependent kinase inhibitors ( $C d k n 1 a$, $C d k n 2 a$ and $C d k n 1 b)^{54}$. EZH2 also phosphorylates retinoblastoma $(\mathrm{Rb})$ protein, which enhances E2F1 release from $\mathrm{Rb}$ and promotes further $\mathrm{EZH} 2$ expression ${ }^{54}$. MYC is transiently induced in B cells that interact with antigen or $\mathrm{T}_{\mathrm{FH}}$ cells. MYC is subsequently downregulated as B cells commit to the GC fate, perhaps via direct transcriptional repression by BCL- 6 (REF. ${ }^{52}$ ).

The transcriptional activators MEF2C and MEF2B also promote GC development ${ }^{55-57}$. MEF2C expression is induced by BCR signalling and regulates the expression of genes important for B cell proliferation 


\section{Class-switched}

A cellular process in which proliferating B cells have rearranged their constant region genes to switch from expressing one class of immunoglobulin to another, without altering their antigen specificity.

Dark zone

The compartment of the germinal centre in which $B$ cells proliferate and undergo cells producing the CXCR4 ligand CXCL12.

Light zone The compartment of the capture antigen presented by follicular dendritic cells and compete to present antigen to follicular helper T cells in order to receive signals necessary for their continued survival, proliferation and differentiation. somatic hypermutation, which contains a network of stromal germinal centre in which B cells

and survival, including $C c n d 2$ and B cell lymphomaextra large $(B c l 2 l 1)^{56,57}$. MEF2B is mutated in cases of diffuse large B cell lymphoma (DLBCL) and follicular lymphoma and directly promotes the expression of BCL-6 $\left(\right.$ REF. $\left.^{58}\right)$. Loss of MEF2B results in partial impairment of GC formation, with MEF2B regulating the expression of a diverse network of BCL-6-dependent and BCL-6-independent genes that are important for GC development and function ${ }^{55}$. MEF2C and MEF2B can form dimers, with ablation of both proteins resulting in a marked reduction in GC development, suggesting that they may have partially redundant functions ${ }^{55}$.

The basic helix-loop-helix transcription factors 3 (TCF3; also known as E2A) and TCF4 function together to promote GC B cell development ${ }^{59,60}$. TCF3 and TCF4 act primarily as transcriptional activators in $\mathrm{B}$ cells and regulate the expression of genes critical for GC development, including Icosl, Mef $2 b$ and Pou $2 a f 1$ (REFS ${ }^{60,61}$ ). TCF 3 and TCF4 are expressed in B cells before activation but have limited activity owing to inhibition by inhibitor of DNA binding 3 (ID3). B cell activation promotes the downregulation of ID3, leading to increased TCF3/TCF4 activity owing to reduced repression by ID3 $\left(\right.$ REF. $\left.^{61}\right)$. Deficiency in ID3 results in reduced GC $\mathrm{B}$ cell development, perhaps owing to aberrant activity of TCF3/TCF4 (REF.62).

The zinc finger transcription factor Yin and Yang 1 (YY1) is also important for the development and maintenance of GC B cell development ${ }^{63}$. YY1 is important for all stages of $B$ cell differentiation and the precise mechanism by which YY1 promotes the GC response is currently unclear. Similarly, the transcription factor early B cell factor 1 (EBF1) is necessary for the development of mature B cell subsets, with deficiency in EBF1 resulting in a reduction in GC B cell development and maintenance ${ }^{64,65}$.

\section{Box 2 | Memory B cell subsets}

Numerous cell surface markers, including CD80, PDL2, CD44, CD62L and CD73, are differentially expressed on memory $B$ cells $(M B C s)^{5,8,142}$. Three major $M B C$ subsets have been defined in the mouse: $\mathrm{CD} 80^{-} \mathrm{PDL2}^{-}$(double negative), $\mathrm{CD} 80^{-} \mathrm{PDL2}^{+}$(single positive) and $\mathrm{CD} 80^{+} \mathrm{PDL}^{+}{ }^{+}$(double positive) ${ }^{5}$. MBC subsets develop during three overlapping periods, with double-positive MBCs developing last and having undergone the greatest amount of somatic hypermutation and class-switching ${ }^{5,6}$. The extent of CD40 signalling may regulate $\mathrm{MBC}$ subset development ${ }^{87}$. The $\mathrm{MBC}$ isotype has also been reported to regulate $\mathrm{MBC}$ function upon recall, with $\lg \mathrm{M}^{+} \mathrm{MBC}$ s preferably developing into germinal centre (GC) $\mathrm{B}$ cells and $\mathrm{lg}^{+} \mathrm{MBC}$ s developing into antibodysecreting cells ${ }^{2,4}$. However, subsequent studies found that subset composition, not isotype, was the determining factor for the $\mathrm{MBC}$ fate upon recall, with double-negative $M B C$ s preferably developing into $G C B$ cells and double-positive $M B C s$ developing into antibody-secreting cells ${ }^{5}$. Single-positive MBCs had an intermediate phenotype and could develop into either GC B cells or antibody-secreting cells $s^{5}$. MBC subsets express distinct transcriptional signatures, which likely regulates their function upon recall ${ }^{5,8}$. Similar murine $\mathrm{MBC}$ subsets have been identified in numerous immune contexts, including following influenza, lymphocytic choriomeningitis virus and malaria infection and during commensal-driven responses in Peyer's patches ${ }^{8,14,20,143,144}$. The relationship between murine and human $\mathrm{MBC}$ subsets remains unclear. Considering that human MBCs express CD80, but not PDL2 or CD73, it appears that human and murine MBC subsets express only partially overlapping markers ${ }^{142,145,146}$. Markers of human MBCs include CD27, CD21, CCR2, CEACAM21, Toll-like receptors (TLRs) and Fc-receptor-like proteins ${ }^{120,147,148}$. An improved understanding of the functional capacities of human $M B C$ subsets will be essential for the design of vaccines that are maximally effective in inducing durable immunity.

\section{Regulation of GC B cell maintenance}

BCL-6 expression is essential for maintenance of the GC state through multiple mechanisms, including regulation of the stability of the transcriptional repressor BTB domain and CNC homology 2 (BACH2) ${ }^{66}$ (FIG. 2). BCL- 6 and $\mathrm{BACH} 2$ cooperate to regulate the GC transcriptional programme and repress expression of plasma cell-defining factors, such as B lymphocyte-induced maturation protein 1 (BLIMP1; also known as PRDM1 $)^{66-68}$. BCR and CD40 signalling can transiently disrupt BCL-6 activity through mitogen-activated protein kinase (MAPK) signalling and post-translational modification of the BCL-6 co-repressors SMRT and $\mathrm{NCOR}^{69,70}$. Sustained CD40 signalling can promote IRF4 expression, which can directly repress BCL-6 expression, resulting in enhanced expression of BCL6-repressed BLIMP1 and, ultimately, plasma cell differentiation ${ }^{36,71,72}$. The $\mathrm{T}_{\mathrm{FH}}$ cell-derived cytokines IL-4 and IL-21 can bind to IL-4R and IL-21R on B cells, and downstream signalling from these receptors counteracts BCR signalling-induced degradation of BCL-6 (REFS $\left.{ }^{29,73}\right)$. GC B cells express the E3 ubiquitin-protein ligase CBL, which promotes IRF4 degradation and prevents premature GC exit ${ }^{74}$. CBL ubiquitin ligases also negatively regulate $\mathrm{BCR}$ signalling through the degradation of SYK $^{75,76}$. Strong CD40 and BCR signalling can lead to a decrease in expression of CBL and facilitate plasma cell differentiation ${ }^{74}$.

There is emerging evidence that the transcription factor requirements for GC responses may differ depending on the type of immune response. For example, T-bet (also known as T-box transcription factor 21 (TBX21)) acts in a B cell-intrinsic manner to both restrict the overall GC response and promote the dark zone GC $B$ cell transcriptional programme following malaria infection ${ }^{77,78}$. T-bet may also be needed for the spontaneous interferon- $\gamma$ (IFN $\gamma$ )-driven GC responses that occur in mice prone to autoimmunity ${ }^{79}$, although this is not universally the case ${ }^{80}$. T-bet ${ }^{+} \mathrm{MBC}$, also known as atypical MBCs, accumulate during autoimmunity, chronic infection and ageing and participate in both protective and pathogenic immune responses (BOX 3).

The transcription factor forkhead box $\mathrm{O} 1$ (FOXO1) is critical for the development of dark zone GC B cells ${ }^{81-83}$. Ablation of FOXO1 leads to an impairment in the dark zone transcriptional programme and reduced affinity maturation. FOXO1 regulates the expression of numerous genes, including Cxcr4, which encode a G-proteincoupled receptor necessary for migration of $B$ cells to the dark zone ${ }^{82,84}$. Transforming growth factor- $\beta$ (TGF $\beta$ ) and IL-10 signalling in GC B cells promote FOXO1 expression $^{85,86}$. The transcriptional programme regulated by FOXO1 largely overlaps with that of BCL-6, suggesting that FOXO1 and BCL- 6 cooperate to support the dark zone state ${ }^{82}$. FOXO1 is important for maintenance of the GC proliferative state and is required for the induction of basic leucine zipper transcription factor (BATF) expression in light zone GC cells. FOXO1 does not directly induce BATF but rather is likely necessary to upregulate the expression of CD40 and BCR signalling components that subsequently promote BATF expression $^{83,87}$. BATF is required for GC maintenance 


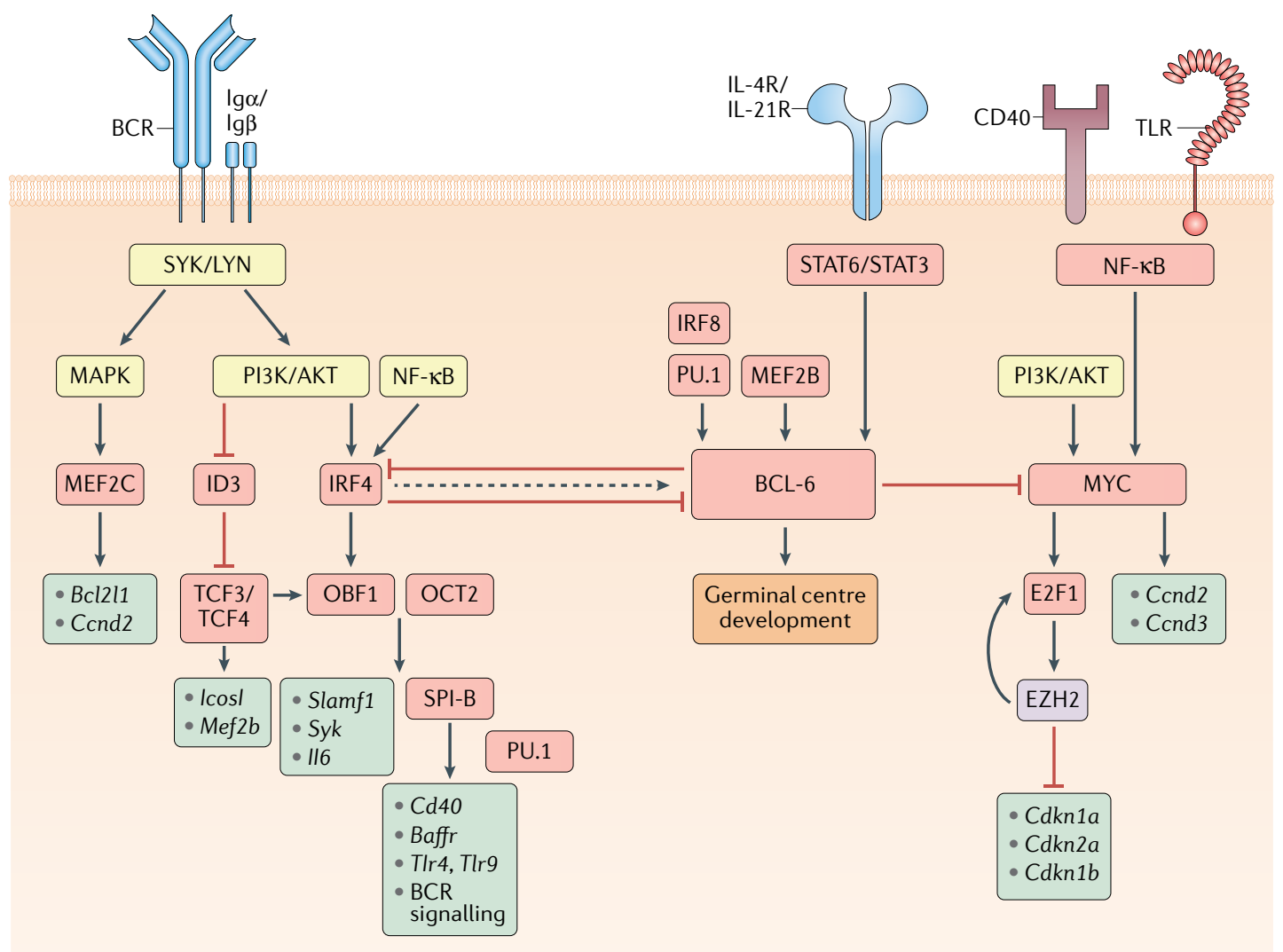

Fig. 1 | Transcriptional regulation of GC B cell commitment. Model for signalling pathways and transcription factors that regulate $B$ cell commitment to the germinal centre (GC) fate. Boxes that indicate signalling molecules are coloured yellow, transcription regulators red, downstream gene targets turquoise and epigenetic modifiers purple. The B cell receptor (BCR), via its signalling subunits $\lg \alpha$ and $\lg \beta$ as well as downstream tyrosine kinases such as SYK and LYN, activates the mitogen-activated protein kinase (MAPK) and phosphoinositide 3-kinase (PI3K)/AKT pathways. MAPK signalling induces the expression of the transcriptional activator myocyte enhancer binding factor 2c (MEF2C), which promotes the transcription of B cell lymphoma-extra large (Bcl2l1) and cyclin D2 (Ccnd2). The expression of the transcriptional repressor inhibitor of DNA binding 3 (ID3) is reduced following B cell activation, allowing for transcription factor 3/4 (TCF3/4)-driven induction of Icosl and Mef2b transcription. PI3K/AKT and nuclear factor- $\mathrm{KB}$ (NF- $\mathrm{kB}$ ) signalling also induce the expression of the transcription factor interferon regulatory factor 4 (IRF4). IRF4 and TCF3/4 induce the expression of the co-activator OBF1 (also known as POU class 2 homeobox associating factor 1 (POU2AF1)), which cooperates with OCT2 (also known as POU class 2 homeobox 2 (POU2F2)) to promote transcription of Slamf1, Syk and 116. OBF1 and OCT2 can also induce the expression of the transcription factor SPI-B, which acts in a redundant fashion with PU.1 to enhance the transcription of genes encoding B cell surface receptors, such as $C d 40$, B cell activating factor receptor (Baffr), Toll-like receptor 4 (Tlr4) and Tlr9, and many components of the BCR signalling pathway, including Blnk and Btk. Although transiently elevated levels of IRF4 can induce the expression of B cell lymphoma 6 (BCL-6), sustained IRF4 levels will repress BCL- 6 expression. BCL-6 expression is also induced by the transcription factors MEF2B and IRF8/ PU.1 as well as the cytokines IL-4 and IL-21, which bind to their respective receptors (IL-4R and IL-21R) and induce signal transducer and activator of transcription 6 (STAT6)/STAT3 signalling. CD40 and/or TLR-driven NF- $\mathrm{kB}$ signalling, alongside $\mathrm{PI} 3 \mathrm{~K} / \mathrm{AKT}$ signalling, will induce the expression of the transcription factor MYC, which promotes cellular proliferation by inducing the transcription of $C \mathrm{cnd} 2 / \mathrm{C} c \mathrm{nd} 3$ and the expression of the transcription factor E2F transcription factor 1 (E2F1). E2F1 induces expression of Ezh2 that encodes a Polycomb repressive complex 2 (PRC2) enzymatic component. Enhancer of zeste homologue $2(\mathrm{EZH} 2)$ promotes cell cycle progression by repressing the expression of $\mathrm{Cdkn1a}, \mathrm{Cdkn2a}$ and $\mathrm{Cdkn} 1 b$, which encode cyclin-dependent kinase inhibitors. $\mathrm{EZH} 2$ also promotes $\mathrm{E} 2 \mathrm{~F} 1$ release from the retinoblastoma $(\mathrm{Rb})$ protein via phosphorylation of Rb, thereby enhancing E2F1 activation and further EZH2 expression. BCL-6 can directly repress MYC expression, thereby limiting the number of cell divisions that GC B cells undergo. BCL-6 promotes GC B cell development through regulation of numerous genes controlling cellular processes including the DNA damage response, B cell migration, apoptosis, BCR and CD40 signalling, plasma cell differentiation and T cell:B cell interactions. Together, these transcriptional regulators allow for the precise control of GC initiation that is necessary to balance the competing needs of the immune system to induce a protective response while limiting immunopathology.

and contributes to the GC proliferative state ${ }^{83}$. BATF also directly regulates the expression and targeting of activation-induced cytidine deaminase (AID) ${ }^{88}$.

Phosphoinositide 3-kinase (PI3K) signalling actively restricts FOXO1 expression in light zone GC B cells, with only a small fraction of light zone GC B cells expressing FOXO1 $\left(\mathrm{REFS}^{81,82}\right)$. FOXO1-expressing light zone GC $B$ cells also express MYC, suggesting that they are actively receiving $\mathrm{T}$ cell help ${ }^{81}$. MYC expression is induced in amounts proportional to the strength of $\mathrm{T}$ cell 
help and determines the number of cell divisions that occur in the dark zone ${ }^{89}$. MYC regulates multiple aspects of cell proliferation including metabolism and the expression of proteins that are necessary to sustain cell division, such as activating enhancer binding protein 4 (AP4; also known as TFAP4) and ubiquitin-like, containing PHD and RING finger domains, 1 (UHRF1) ${ }^{89,90}$. MYC interacts with the transcriptional activator
MYC-interacting zinc finger protein 1 (MIZ1) to form a transcriptional repressor complex that represses MIZ1 target genes ${ }^{91}$. The MYC-MIZ1 complex promotes cell cycle entry of GC B cells actively receiving T cell help ${ }^{91}$. Whereas MYC is rapidly downregulated in GC B cells that enter the dark zone, AP4 expression is maintained and is necessary for dark zone B cells to undergo continued cell division ${ }^{92}$.

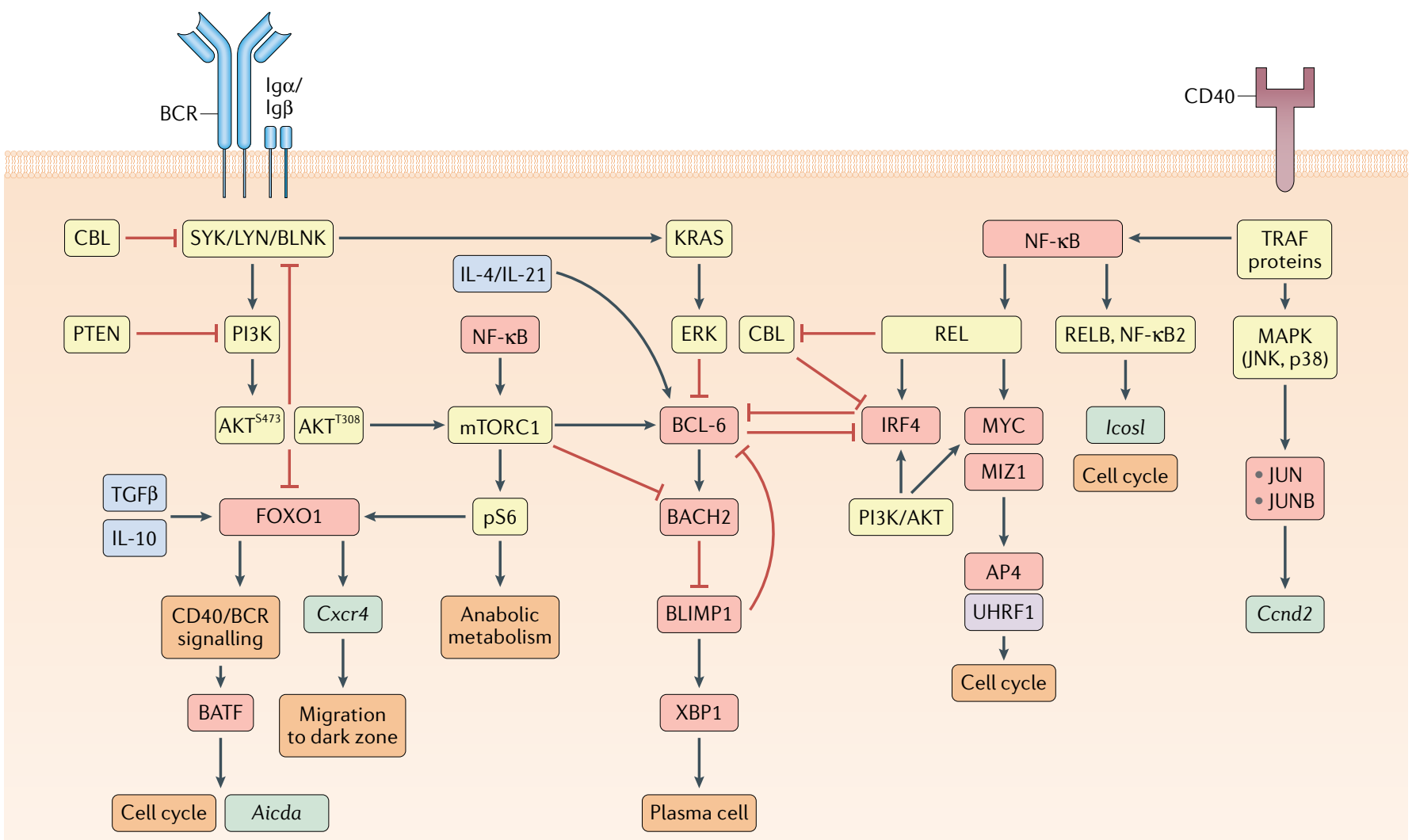

Fig. 2 | Transcriptional regulation of GC B cell maintenance. Model for signalling pathways and transcription factors that regulate germinal centre (GC) B cell maintenance. Boxes that indicate signalling molecules are coloured yellow, transcription factors red, downstream gene targets turquoise, cytokines blue (drawn inside cell for simplicity) and epigenetic modifiers purple. The $B$ cell receptor (BCR), via its signalling subunits lg $\alpha$ and $\lg \beta$, engages the tyrosine kinases SYK and LYN and the adapter protein BLNK, leading to activation of phosphoinositide 3-kinase (PI3K) and subsequent phosphorylation of AKT at S473. In GC B cells, AKT is already phosphorylated at T308. PI3K/AKT signalling represses forkhead box O1 (FOXO1) expression and attenuates BCR signalling through phosphorylation of the signalling molecules SHP1, CSK and HPK1, which negatively regulate the activity of SYK, LYN and BLNK, respectively. GC B cells also express high levels of the phosphatase and tensin homologue (PTEN), which restrains PI3K-mediated phosphorylation of AKT at S473. PI3K/AKT signalling, along with $\mathrm{CD} 40$-driven nuclear factor- $\mathrm{KB}(\mathrm{NF}-\mathrm{\kappa B})$ activity, can induce mechanistic target of rapamycin complex 1 (mTORC1) signalling and lead to the phosphorylation of the ribosomal protein $\mathrm{S} 6(\mathrm{pS} 6)$ and the adoption of an anabolic metabolic state through the increased accumulation of biomass. mTORC 1 can also promote the expression of $B$ cell lymphoma 6 (BCL-6) and repress $\mathrm{BTB}$ domain and $\mathrm{CNC}$ homology 2 (BACH2) expression. $\mathrm{pS6}$, alongside IL-10 and transforming growth factor- $\beta$ (TGF $\beta$ ), can induce the expression of the transcription factor FOXO1. FOXO1 promotes the transcription of $C x c r 4$, which facilitates GC B cell migration to the dark zone. FOXO1 also promotes $C D 40 / B C R$ signalling, indirectly resulting in expression of the transcription factor basic leucine zipper transcription factor (BATF). BATF directly promotes the transcription of Aicda and cell cycle progression, likely through interaction with other transcription factors of the JUN or interferon regulatory factor (IRF) families. BCR signalling also engages the GTPase KRAS, which activates the kinase ERK, resulting in repressed BCL-6 expression. CD40 signalling leads to the recruitment of the kinase MEKK1 to the adapter molecule TRAF2 and results in activation of the mitogen-activated protein kinases (MAPKs) JNK and p38. MAPK signalling induces the expression of the transcription factors JUN and JUNB, as well as the transcription of Ccnd2. CD40 signalling also signals through TRAF proteins to induce NF- $\kappa B$. $N F-\kappa B$ signals through both the canonical (REL) and non-canonical (RELB, $\mathrm{NF}-\kappa \mathrm{B} 2$ ) pathways to regulate GC maintenance. RELB and NF- $\mathrm{KB} 2$ induce Icosl expression and promote the induction of the cell cycle. The precise

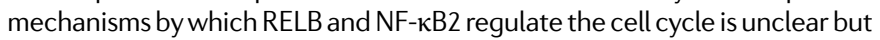
may relate to induction of ICOSL expression. REL and PI3K/AKT signalling induce the expression of IRF4 and MYC. MYC acts in a complex with MYC-interacting zinc finger protein 1 (MIZ1) to induce the expression of activating enhancer binding protein 4 (AP4) and the epigenetic regulator ubiquitin-like, containing PHD and RING finger domains, 1 (UHRF1), both of which promote the cell cycle. Strong canonical NF- $\kappa B$ signalling also represses expression of the ubiquitin ligase CBL (not shown). CBL inhibits plasma cell differentiation through repression of IRF4 expression (not shown) and BCR signalling via degradation of SYK. IRF4 and BCL-6 are mutually antagonistic transcription factors. BCL-6 induces $\mathrm{BACH} 2$ expression, which represses $B$ lymphocyte-induced maturation protein 1 (BLIMP1) expression. In turn, BLIMP1 can repress BCL-6 expression and induce XBP1 expression, resulting in plasma cell differentiation. 


\section{Box 3 | Atypical memory B cells}

$B$ cells expressing T-bet, CD11c, CD11b and/or FCRL 5 develop in many individuals with chronic infection or autoimmune disease and are often referred to as atypical memory $B$ cells (MBCs). Atypical MBCs are a heterogeneous population of antigen-experienced cells that are dependent on $\mathrm{CD} 4^{+} \mathrm{T}$ cells and IL-21 for their development ${ }^{149,150}$. Toll-like receptor 7 (TLR7) ligand and interferon- $\gamma$ (IFN $\gamma$ ) stimulation also promote atypical MBC development ${ }^{151}$. Plasmodium-specific MBCs develop in the absence of germinal centres (GCs) and mature follicular helper T cells, suggesting that GCs may not be required for atypical MBC development ${ }^{20}$. Atypical MBCs can persist long term and are associated with both protective and pathogenic immune responses ${ }^{14,152}$. Expansion of atypical $\mathrm{MBC}$ s is also associated with progression of autoimmune diseases ${ }^{79}$. Atypical MBCs expand following infection or protein immunization and rapidly differentiate into GC $B$ cells or antibody-secreting cells upon recall ${ }^{14,21,153}$. Atypical MBCs can be either $\lg M^{+}$ or class-switched, with the expression of T-bet important for the development of $\operatorname{lgG} 2 \mathrm{a}^{+}$ cells ${ }^{154}$. T-bet expression also distinguishes MBC subsets with distinct homing and functional properties ${ }^{155}$. The transcription factor MYB represses T-bet expression in $B$ cells ${ }^{156}$. Whereas $B$ cell intrinsic expression of T-bet in MBCs is required for plasma cell differentiation and protection following viral challenge, T-bet is not required for the development of atypical MBCs ${ }^{157,158}$. Future studies investigating the impact of atypical MBC depletion on the development of autoimmune disease will be important in assessing the attractiveness of atypical MBCs as a therapeutic target.

Signals downstream of CD40 and the BCR synergistically induce MYC expression in GC B cells ${ }^{93}$. CD40 signalling activates the transcription factor NF- $\kappa \mathrm{B}$ but does not activate PI3K, which is instead induced by BCR signalling ${ }^{93}$. CD40 signalling can also induce the expression of MAPKs such as JNK and p38, which promote the GC response by inducing expression of the transcription factors JUN and JUNB ${ }^{94}$. Canonical NF- $\kappa B$ signalling drives activation of the NF- $\kappa B$ subunits REL and RELA (also known as p65) ${ }^{95}$. REL is required for GC B cell maintenance, with ablation of REL resulting in GC collapse owing to a failure to sustain the metabolic programme necessary for cell growth ${ }^{95}$. REL can induce IRF4 and MYC expression in B cells ${ }^{96,97}$. Non-canonical NF- $\kappa \mathrm{B}$ signalling also promotes GC maintenance, and experimental deletion of the alternative NF- $\kappa B$ subunits RELB and NF- $\kappa B 2$ result in a reduced GC response $^{98}$. Non-canonical NF- $\kappa B$ signalling acts independently of REL to promote cell proliferation and the expression of ICOSL in light zone GC B cells ${ }^{98}$. BCR signalling is generally attenuated in GC B cells owing to negative feedback resulting from increased expression of phosphatase and tensin homologue (PTEN) and phosphoinositide-dependent kinase 1 (PDK1) ${ }^{99}$. However, BCR signalling can sufficiently overcome this attenuation to signal through $\mathrm{PI} 3 \mathrm{~K}$ and restrict FOXO1 activity in a protein kinase B (also known as AKT)-dependent manner ${ }^{93,100}$.

$\mathrm{T}$ cell help activates mechanistic target of rapamycin complex 1 (mTORC1) signalling and triggers GC B cells to undergo the anabolic cell growth necessary to sustain rapid cell division ${ }^{101,102}$. mTORC1 activity is dependent on the phosphorylation of the ribosomal protein S6 (pS6) $)^{101}$. Both BCR and CD40 signalling are necessary for strong phosphorylation of S6 by mTORC1 (REF $\left.{ }^{93}\right)$. mTORC1 is also important for the induction of BCL- 6 expression and repression of $\mathrm{BACH} 2$ expression in $\mathrm{B}$ cells ${ }^{102,103}$. Inhibition of mTOR signalling via rapamycin results in a failure to upregulate FOXO1 expression ${ }^{101}$. Considering that PI3K signalling both promotes mTORC1 and restricts FOXO1, it is unclear why mTORC1 would be necessary for FOXO1 induction ${ }^{81,82}$. One possibility is that there is temporal separation between the initial BCR signal that represses FOXO1 and the subsequent CD40 signalling that induces mTORC1, which allows sufficient time for FOXO1 to regain activity before migration to the dark zone. Initial repression of FOXO1 would also allow GC B cells that had received a BCR signal to engage with T cells in the light zone and upregulate MYC before migration to the dark zone.

\section{Regulation of MBC differentiation}

Low-affinity light zone GC B cells generally undergo apoptosis owing to a failure to receive $\mathrm{T}$ cell help ${ }^{104,105}$ (FIC. 3). GC B cells that receive low levels of T cell help have low activation of mTORC1 and display a reduced accumulation of biomass relative to cells that had received $\mathrm{T}$ cell help ${ }^{101}$. These cells also fail to express MYC, which is required for both the accumulation of metabolites necessary for cell division and for entry into the cell cycle ${ }^{89}$. MYC functions in complex with MIZ1 to promote plasma cell development and restrict $\mathrm{MBC}$ differentiation ${ }^{91}$. GC B cells that receive low levels of $\mathrm{T}$ cell help also maintain elevated $\mathrm{BACH} 2$ expression, perhaps owing to reduced repression of $\mathrm{BACH} 2$ transcription by mTORC1 (REFS ${ }^{103,106}$ ). BACH2 expression predisposes GC B cells to differentiate into $\mathrm{MBCs}^{7}$. The precise mechanisms by which $\mathrm{BACH} 2$ functions to promote $\mathrm{MBC}$ development remain to be elucidated but might relate to repression of cyclin-dependent kinase inhibitor genes (Cdkn1a,Cdkn2a) and the induction of the anti-apoptotic gene $B c l 2 l 1^{107}$. Expression of prosurvival genes, such as $B c l 2$, and repression of pro-apoptotic genes facilitate MBC differentiation ${ }^{108,109}$. Together, these studies suggest a model where low levels of $\mathrm{T}$ cell help promote $\mathrm{MBC}$ differentiation by limiting $\mathrm{MTORC} 1$ and MYC-driven cell growth and cell cycle progression, thereby promoting cell survival.

Precursor memory (PreMem) B cells have been identified in the GCs of both mice and humans ${ }^{110-112}$. PreMem B cells tend to localize at the edge of the light zone and they transcriptionally and functionally resemble MBCs, despite expressing GC B cell surface markers. PreMem B cells are somatically mutated and have recently undergone cell division, indicating that they are mature GC B cells that are in the process of differentiating into MBCs. It is likely necessary for PreMem $\mathrm{B}$ cells to exit the antigen-rich light zone to complete their differentiation into MBCs. Downregulation of BCL-6 is important in allowing PreMem B cells to efficiently exit the GC. BCL- 6 induces the expression of the GC-confinement factor S1PR2 and represses the expression of pro-migratory receptors that are likely to be involved in GC exit, such as EBI2 and S1PR1 $\left(\mathrm{REF}^{23}\right)$. BCL-6 also promotes GC B cell apoptosis through repression of BCL-2 expression ${ }^{26}$. Loss of the BCL-6 transcriptional programme is one of the main drivers of the PreMem $B$ cell gene signature ${ }^{110}$.

The finding that BCL- 6 represses MBC differentiation is consistent with a model in which a low level of T cell help favours MBC differentiation ${ }^{113}$. GC B cells that receive low levels of $\mathrm{T}$ cell help have less exposure to the 
$\mathrm{T}_{\mathrm{FH}}$ cell-derived cytokines IL-4 and IL-21, which would normally serve to counteract BCR signalling-mediated degradation of BCL-6 $\left(\mathrm{REF}^{29}\right)$. Considering that BCL-6 and $\mathrm{BACH} 2$ cooperate to regulate the GC B cell transcriptional programme, it will be important to understand the distinct roles of $\mathrm{BACH} 2$ and $\mathrm{BCL}-6$ in promoting $\mathrm{MBC}$ differentiation ${ }^{66}$. Both PreMem $\mathrm{B}$ cells and $\mathrm{MBC}$ express lower levels of $\mathrm{BACH} 2$ relative to bulk GC B cells, suggesting that $\mathrm{BACH} 2$ alone is not sufficient to induce MBC fate commitment ${ }^{110-112}$.

a Weak BCR signalling + weak $T$ cell help

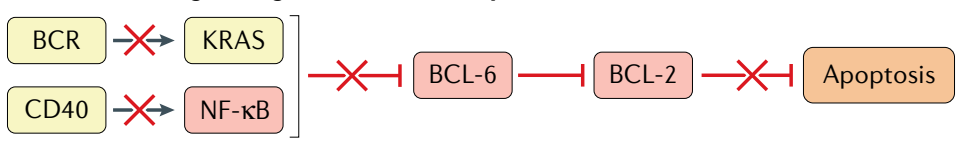

b Weak $T$ cell help

C Intermediate T cell help

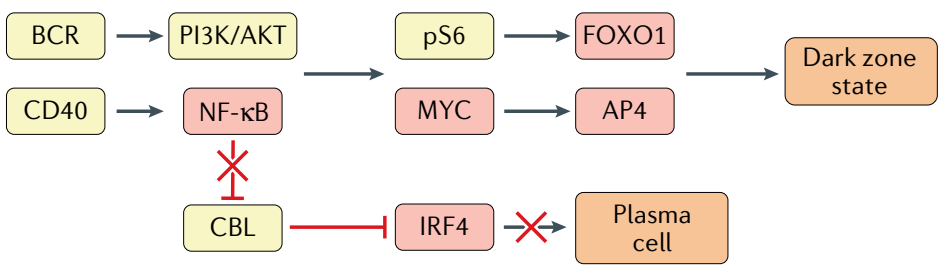

d Strong $\mathrm{T}$ cell help

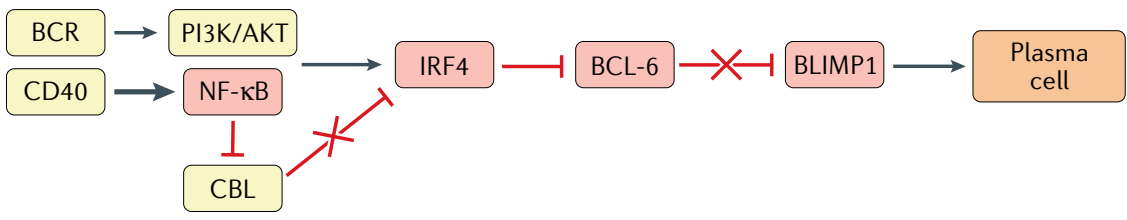

Fig. 3 | Transcriptional regulation of GC B cell differentiation. Germinal centre (GC) B cell differentiation is regulated by the extent of signals received by the $B$ cell through the antigen-engaged B cell receptor (BCR) and CD40 (via CD40Lexpressing T cells). Boxes indicating signalling molecules are coloured yellow, transcription factors red, cytokines blue and downstream gene targets turquoise. A red cross indicates a pathway that is not functional under the condition illustrated. a | Weak BCR signalling and weak $T$ cell help results in a failure of $G C B$ cells to receive sufficient signals through the BCR and CD40 to induce KRAS and nuclear factor- $\mathrm{KB}(\mathrm{NF}-\kappa \mathrm{B})$-driven repression of $\mathrm{B}$ cell lymphoma 6 (BCL-6) expression. Repression of BCL-2 expression by BCL-6 in these cells will result in apoptosis owing to a failure to receive sufficient survival signals. b| GC B cells that receive weak T cell help (transmitted via CD40) will not receive sufficient CD40-induced $\mathrm{NF}-\kappa \mathrm{B}$ signals to indirectly repress $\mathrm{BTB}$ domain and $\mathrm{CNC}$ homology 2 (BACH2). $\mathrm{BACH} 2$ promotes $\mathrm{GC}$ B cell survival through induction of the anti-apoptotic gene Bcl2l1 and repression of cyclin-dependent kinase inhibitor family genes (Cdkn1a, Cdkn2a). BACH2 also represses B lymphocyte-induced maturation protein 1 (BLIMP1) and, accordingly, restricts plasma cell differentiation. The continued survival of $B$ cells that receive weak $T$ cell help is dependent on receiving sufficient BCR-driven KRAS signalling to repress BCL-6 expression. Exposure to T cell-derived IL-4 and IL-21 normally stabilizes BCL-6 expression in GC B cells. However, B cells that receive weak T cell-derived CD40 will also have low exposure to IL-4 and IL-21, resulting in impaired BCL-6 expression and induction of BCL-6-repressed genes such as haematopoietically expressed homeobox (Hhex) and Bcl2. HHEX interacts with the co-repressor transducin-like enhancer 3 (TLE3) to further repress BCL-6 expression and promote memory B cell development through the upregulation of BCL-2 and the transcription factor Sloan-Kettering Institute (SKI). c | GC B cells that receive intermediate T cell help receive both phosphoinositide 3-kinase (PI3K)/AKT and NF- $\mathrm{kB}$ signalling, resulting in phosphorylation of the ribosomal protein S6 (pS6) and expression of MYC. pS6 and MYC subsequently induce expression of forkhead box O1 (FOXO1) and activating enhancer binding protein 4 (AP4), respectively, ultimately leading to the adoption of the highly proliferative dark zone state. Intermediate levels of CD40 signalling do not drive sufficient NF- $\mathrm{BB}$ signalling to repress expression of the ubiquitin ligase CBL, allowing CBL to continue to repress interferon regulatory factor 4 (IRF4) expression and prevent plasma cell differentiation. $\mathbf{d}$ | GC B cells that receive strong T cell help receive both PI3K/AKT and NF- $\kappa B$ signalling. Strong NF- $\kappa B$ signalling represses $C B L$ expression and allows for upregulation of IRF4. IRF4 represses BCL-6 expression and leads to expression of BLIMP1 and plasma cell differentiation. 
The transcription factor haematopoietically expressed homeobox (HHEX) has recently been identified as an important promoter of $\mathrm{MBC}$ differentiation. Ablation of Hhex in GC B cells leads to a marked decrease in the development of PreMem cells and $\mathrm{MBCs}^{8}$. BCL- 6 directly represses the expression of HHEX in GC B cells ${ }^{8,114}$. Ablation of Hhex leads to increased expression of BCL- 6 and reduced expression of the BCL- 6 target gene $B c l 2$ (REFS ${ }^{8,26}$ ). Overexpression of BCL-2 is sufficient to rescue MBC differentiation in Hhex-deficient cells ${ }^{8}$. HHEX also functions to promote MBC differentiation through BCL-2-independent mechanisms. For example, HHEX promotes the expression of the transcriptional cofactor Sloan-Kettering Institute (SKI), with overexpression of SKI sufficient to rescue MBC development in the absence of $H h e x^{8}$. The promotion of MBC development by SKI might relate to the observation that retroviral overexpression of SKI imparts a proliferative advantage to CD40-engaged $\mathrm{B}$ cells in vitro ${ }^{115}$. Overexpression of the transcription factor Krüppel-like factor 2 (KLF2) also provides a competitive advantage in vitro and modestly promotes MBC differentiation ${ }^{8,115}$.

Ablation of transducin-like enhancer 3 (Tle3), which encodes a transcriptional co-repressor, in GC B cells also leads to a marked reduction in MBC differentiation ${ }^{8}$. TLE3 does not directly bind to DNA but rather functions through interaction with DNA-binding transcription factors and subsequent recruitment of histone deacteylases (HDACs), which mediate gene repression ${ }^{116,117}$. TLE3 can directly interact with HHEX via an EH-1 motif and mutation of this motif is sufficient to prevent HHEX from promoting MBC development ${ }^{8}$. TLE family members interact with numerous transcription factors, including TCF1, lymphoid enhancer-binding factor 1 (LEF1) and runt-related transcription factor 1 (RUNX1) and RUNX3, to regulate cell differentiation ${ }^{116}$. Ablation of Runx3 in GC B cells leads to a slight increase in $\mathrm{MBC}$ differentiation, suggesting that competition for TLE3 binding between HHEX and other transcription factors might serve as a mechanism for regulating $\mathrm{MBC}$ differentiation ${ }^{8}$.

HHEX is not required for MBC maintenance but can regulate the ability of $\mathrm{MBCs}$ to respond upon antigen re-encounter in certain immune settings ${ }^{8}$. IL-9R signalling is also a regulator of $\mathrm{MBC}$ recall upon antigen re-encounter ${ }^{118}$. Ablation of $I L-9 R$ results in attenuated recall antibody responses to $\mathrm{T}$ cell-dependent antigens. $I L-9 R$-deficient MBCs had augmented ability to differentiate into GC B cells upon recall. IL-9R is not required for $\mathrm{MBC}$ maintenance, with contrasting data on whether IL-9 acts to promote MBC differentiation ${ }^{111,118}$. IL-9 is produced by MBCs and a very small proportion of $\mathrm{T}_{\mathrm{FH}}$ cells and can signal through STAT3 and STAT5 $\left(\right.$ REFS $\left.^{111,118}\right)$. MBCs also express the transcription factor zinc finger and BTB-containing 32 (ZBTB32), which acts to negatively regulate the magnitude and duration of the antibody recall response through regulation of the cell cycle and mitochondrial function ${ }^{119}$. The increased expression of $\mathrm{ZBTB} 32$ in $\mathrm{CD} 80^{+} \mathrm{PDL} 2^{+} \mathrm{MBCs}$, relative to $\mathrm{CD} 80^{-} \mathrm{PDL} 2^{-} \mathrm{MBCs}$, might help regulate the differential fate of these subsets upon secondary challenge.
Numerous genes besides $Z b t b 32$, including $T l r 7$ and $T l r 9$, are differentially expressed between MBC subsets ${ }^{5}$. How differences in naive $\mathrm{B}$ cell and $\mathrm{MBC}$ responsiveness to inputs such as TLR, CD40 and BCR signalling determine the relative ability of these cells to proliferate and differentiate upon reactivation remains to be fully understood $^{120-122}$.

\section{Models of MBC development}

The asymmetric fate model. Multiple models of MBC development have been proposed ${ }^{123,124}$. The asymmetric fate model suggests that MBCs arise in a stochastic manner from GC B cells. This is supported by data from some animal models showing that a genetically induced decline in GC B cells results in a proportional loss in $\mathrm{MBCs}^{125}$ (FIG. 4a). It is thought that GC B cells that interact with $\mathrm{T}_{\mathrm{FH}}$ cells can undergo asymmetric cell division, resulting in an unequal distribution of cell fate-determining molecules such as BCL-6, IRF4 and IL-21R among their cell progeny ${ }^{126,127}$. Daughter cells that inherit IRF4 will preferentially differentiate into plasma cells, whereas cells that inherit BCL-6 and IL-21R will maintain GC B cell identity or differentiate into MBCs. However, in vitro work with activated B cells and mathematical modelling studies suggest that GC $B$ cells generally undergo symmetric cell division, calling into question the functional relevance of asymmetric cell division in the regulation of MBC differentiation ${ }^{123,128}$.

The instructive fate model. The instructive fate model posits that MBC development is actively regulated by cell-extrinsic signals, such as cytokines and cell contactdependent signals (FIG. 4b). This model is supported by the finding that perturbations in the ability of GC $\mathrm{B}$ cells to receive $\mathrm{T}$ cell help (for example, an inability to sense IL-21 or migrate to the dark zone) results in an accumulation of MBCs that is disproportionate to GC $\operatorname{size}^{28,30,84}$. A central mechanism underlying the instructive fate model is antigen affinity, as higher affinity GC $\mathrm{B}$ cells will receive stronger $\mathrm{T}$ cell help, which promotes the expression of plasma cell-defining transcription factors such as IRF4 and BLIMP1 (REF. ${ }^{123}$ ). The instructive fate model is still probabilistic as cells with the same antigen affinity can have different fates, with the affinity influencing the probability of a particular fate ${ }^{123,129}$. The probabilistic nature of the GC likely facilitates the differentiation of high-affinity GC B cells into MBCs and is reflected in the widely disparate rates at which individual GCs lose clonal diversity ${ }^{112,130}$. However, the instructive fate model does not directly account for the observation that MBCs tend to emerge from the GC before plasma cells ${ }^{6-8}$.

The decreasing-potential fate model. The decreasingpotential fate model posits that the cumulative signals received by GC B cells regulate cell fate (FIG. 4C). During the early GC reaction, $B$ cells will have undergone fewer rounds of cyclic re-entry and will have received less cumulative $\mathrm{T}$ cell help. This reduced overall selection signal will predispose early GC B cells to develop into MBCs. As the GC response matures, the cumulative $\mathrm{T}$ cell help received by GC B cells will also increase, 
a Asymmetric fate model

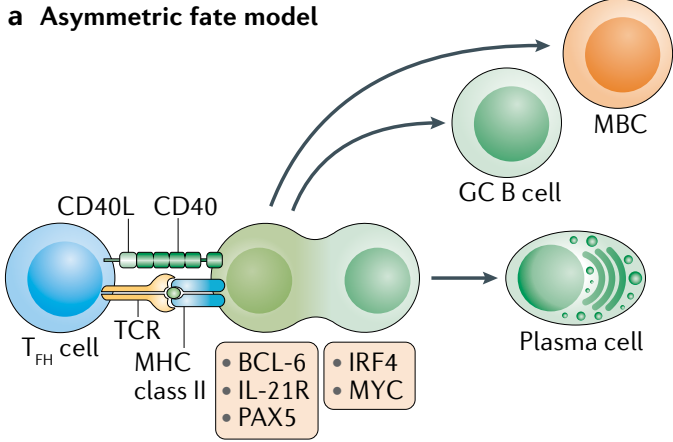

C Decreasing-potential fate model

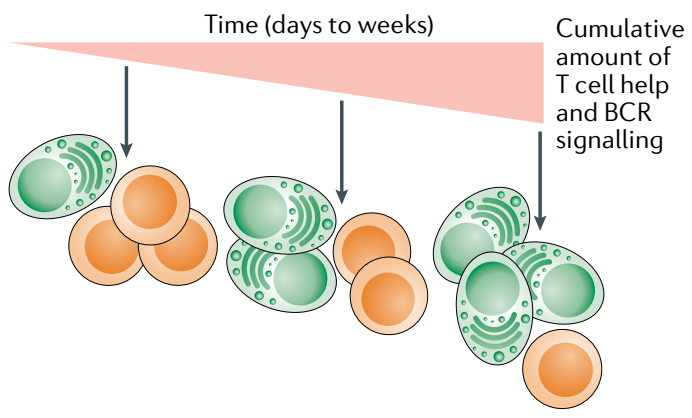

b Instructive fate model

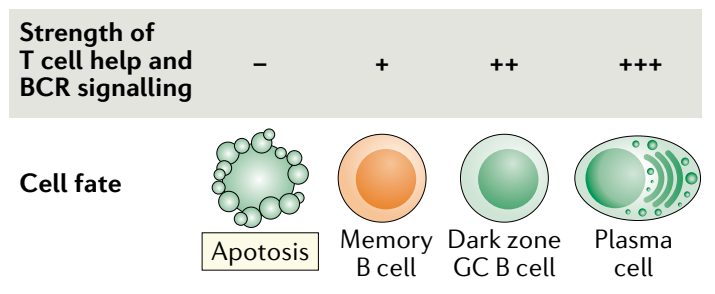

d Integrative fate model

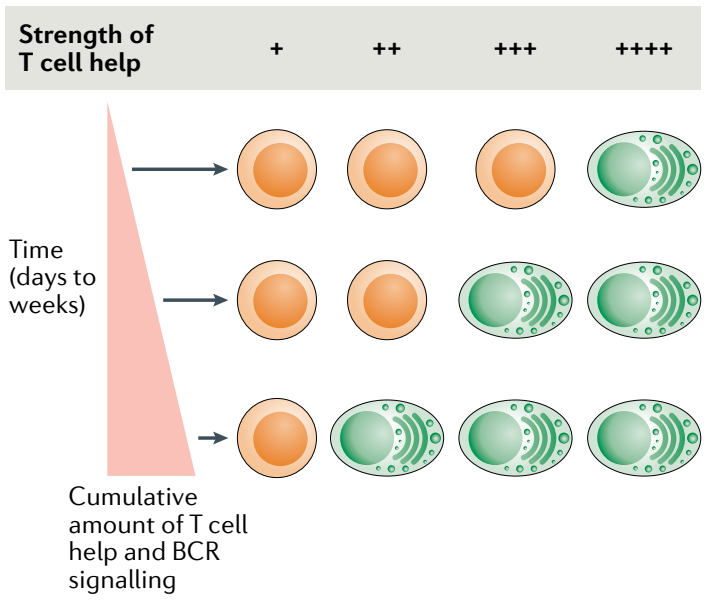

Fig. 4 | Models of GC B cell differentiation. a | Asymmetric fate model. Germinal centre (GC) B cells that interact with follicular helper $\mathrm{T}\left(\mathrm{T}_{\mathrm{FH}}\right)$ cells will undergo cellular polarization, resulting in unequal distribution of fate-altering molecules by daughter cells. Daughter cells inheriting fate-altering molecules that promote the expression of interferon regulatory factor 4 (IRF4) and MYC preferentially differentiate into plasma cells, whereas cells inheriting B cell lymphoma 6 (BCL-6), PAX5 and IL-21R either maintain GC B cell identity or differentiate into memory B cells (MBCs). $\mathbf{b}$ | Instructive fate model. The strength of T cell help and B cell receptor (BCR) signalling received by GC B cells dictates cell fate. Strong T cell help favours plasma cell differentiation and the dark zone state, whereas weak T cell help predisposes GC B cells to differentiate into MBCs or to undergo apoptosis. $\mathbf{c}$ | Decreasing-potential fate model. GC B cell fate is determined by the cumulative strength of T cell help and BCR signalling, accrued over the length of duration in the GC (days to weeks). As GC $B$ cells undergo repeated rounds of cyclic re-entry, they progressively lose their ability to differentiate into MBCs, resulting in a shift towards increased plasma cell differentiation at late GC time points. $\mathbf{d}$ | Integrative fate model. GC B cells integrate both the current quality and the cumulative amounts of received signals when making cell fate decisions. GC B cells that have received less cumulative $T$ cell help and BCR signalling need a stronger signal to differentiate into plasma cells. Conversely, GC B cells that have been in the GC longer and received more cumulative signals require a weaker signal to induce plasma cell differentiation. CD40L, CD40 ligand; MHC, major histocompatibility complex; TCR, T cell receptor.

Type 2 immune responses An immune response that typically occurs in response to extracellular bacteria, parasites, toxins or allergens. Generally characterized by the production of IL-4, IL-5 and IL-13. leading to a shift in GC output towards increased plasma cell differentiation. The decreasing-potential fate model is supported by the observation that early and late GC $B$ cells are transcriptionally and functionally distinct ${ }^{6}$. The precise mechanisms underlying this model remain to be elucidated.

The integrative fate model. The instructive fate model and the decreasing-potential fate model are not mutually exclusive. It is possible that GC B cells integrate both the current quality and the cumulative amounts of received signals when making cell fate decisions (FIG. 4 d). The integrative fate model postulates that, although GC B cells are predisposed to certain fates based on their signalling history, the ultimate cell fate is still influenced by the quality of $\mathrm{T}$ cell help received before differentiation. Therefore, the shift in GC output that occurs over the course of the
GC response may be partially a result of changes in the quality of $\mathrm{T}$ cell help. This is supported by the finding that $\mathrm{T}_{\mathrm{FH}}$ cells change their pattern of cytokine production and surface ligand expression over the course of the GC response. Late $\mathrm{T}_{\mathrm{FH}}$ cells express elevated levels of IL-4 and CD40L and preferentially induce the development of plasma cells during type 2 immune responses ${ }^{73}$. It remains to be determined whether changes in the number and phenotype of $\mathrm{FOXP}^{+}$regulatory $\mathrm{T}$ cells present in the GC could also impact GC output.

\section{Concluding remarks and perspective}

Significant progress has been made towards understanding the signals and transcription factors regulating GC B cell differentiation. However, there remain many knowledge gaps that limit our ability to harness MBCs therapeutically and that need to be addressed in 
future studies. For example, it is unclear whether there are signals that selectively promote MBC differentiation. It is possible that signals that repress BCL- 6 expression without inducing IRF4 expression are sufficient to allow MBC differentiation in GC B cells that survive long enough to express HHEX. However, this model does not account for how factors such as TLE3, which promote MBC differentiation and are not known BCL- 6 targets, are expressed. Similarly, this model does not account for why MBCs tend to develop earlier in the GC response. It will be important to investigate whether the epigenetic state of GC B cells changes over the course of the GC response and limits the ability of GC B cells to differentiate into MBCs at later time points. It will also be important to further explore the extent to which changes in the density and functional properties of $\mathrm{GC}_{\mathrm{FH}}$ cells and $\mathrm{FOXP}^{+} \mathrm{T}$ cells that occur over time regulate $\mathrm{B}$ cell fate decisions.

A better understanding of the functional heterogeneity of MBCs will also be important moving forwards. The MBC population comprises numerous subsets with unique transcriptional profiles and developmental pathways. For example, MBCs can establish residence in mucosal tissues such as the lungs following viral infection and contribute to protection upon pulmonary challenge ${ }^{131,132}$. It is likely that the transcriptional circuitry governing $\mathrm{MBC}$ development will differ between $\mathrm{MBC}$ subsets, with unique transcription factors required for migration to and maintenance within different anatomical sites. Understanding these differences will be critical for the development of therapeutics that can modulate MBC differentiation to overcome the bottleneck in MBC recall responses and to specifically induce the population best equipped to combat a particular pathogen ${ }^{16}$.

Another key emerging area is the study of MBC differentiation in contexts beyond infectious disease. MBCs may contribute to the pathogenesis of certain subtypes of follicular lymphoma and DLBCL. The $\mathrm{t}(14 ; 18)$ translocation is a hallmark of follicular lymphoma and results in constitutive activation of BCL-2. BCL-2 overexpression predisposes B cells to develop into $\mathrm{MBCs}^{109}$. BCL-2-overexpressing MBCs appear to be capable of re-entering the GC multiple times, accruing further mutations and spreading to distant lymphoid organs ${ }^{133}$. These data suggest that MBCs possessing driver mutations might migrate from the primary lymphoma to extra-nodal sites where they accrue further malignant mutations. This process may also be relevant to the MCD/cluster 5 subtype of DLBCL (defined based on co-occurence of $M y d 88^{\mathrm{L} 265 \mathrm{P}}$ and $C d 79 b$ mutations), which has extensive extra-nodal involvement and has one of the lowest survival rates of DLBCL subtypes ${ }^{134,135}$. MBCs were recently found to promote the progression of $\mathrm{MCD} /$ cluster $5 \mathrm{DLBCL}^{136}$. A more precise understanding of the pathways that regulate MBC development and survival could offer new opportunities for the design of therapeutics capable of limiting follicular lymphoma and MCD-DLBCL progression.

MBCs also contribute significantly to disease pathology in individuals with allergy. High-affinity allergen-specific IgG1 ${ }^{+} \mathrm{MBC}$ arise from repeated allergen encounters and can sequentially isotype switch and differentiate into long-lived $\operatorname{IgE}^{+}$plasma cells capable of driving disease pathology ${ }^{137,138}$. IL-13-producing $\mathrm{T}_{\mathrm{FH}}$ cells appear to have a role in the development of high-affinity IgE-producing cells following allergen exposure but not helminth infection ${ }^{139}$. This raises the intriguing notion that there are also unique pathways regulating the differentiation of allergen-specific MBCs. The identification of such pathways would afford the opportunity to selectively target allergen-specific MBC differentiation.

\section{Published online 6 October 2020}

1. Victora, G. D. et al. Germinal center dynamics revealed by multiphoton microscopy with a photoactivatable fluorescent reporter. Cell 143, 592-605 (2010).

2. Dogan, I. et al. Multiple layers of B cell memory with different effector functions. Nat. Immunol. 10, 1292-1299 (2009).

3. McHeyzer-Williams, L. J., Milpied, P. J., Okitsu, S. L. \& McHeyzer-Williams, M. G. Class-switched memory B cells remodel BCRs within secondary germinal centers. Nat. Immunol. 16, 296-305 (2015).

4. Pape, K. A., Taylor, J. J., Maul, R. W., Gearhart, P. J. $\&$ Jenkins, M. K. Different B cell populations mediate early and late memory during an endogenous immune response. Science 331, 1203-1207 (2011).

5. Zuccarino-Catania, G. V. et al. CD80 and PD-L2 define functionally distinct memory $B$ cell subsets that are independent of antibody isotype. Nat. Immunol. 15, 631-637 (2014).

This study identifies new markers that could identify transcriptionally distinct MBC subsets with differing capacities to differentiate into antibodysecreting cells or GC B cells upon antigen re-encounter.

6. Weisel, F. J., Zuccarino-Catania, G. V., Chikina, M. \& Shlomchik, M. J. A temporal switch in the germinal center determines differential output of memory $B$ and plasma cells. Immunity 44, 116-130 (2016). This paper uses a pulse-labelling approach to show that MBC export from the GC precedes that of plasma cells.

7. Shinnakasu, R. et al. Regulated selection of germinalcenter cells into the memory B cell compartment. Nat. Immunol. 17, 861-869 (2016). This study proposes an instructive model of MBC development in which weak $\mathrm{T}$ cell help predisposes
GC B cells to express BACH2 and enter the memory compartment.

8. Laidlaw, B. J., Duan, L., Xu, Y., Vazquez, S. E. \& Cyster, J. G. The transcription factor Hhex cooperates with the corepressor Tle3 to promote memory B cell development. Nat. Immunol 21, 1082-1093 (2020). Using CRISPR-Cas9 screening in vivo, this study found that HHEX and TLE3 cooperate to promote MBC development during a viral response.

9. Purtha, W. E., Tedder, T. F., Johnson, S. Bhattacharya, D. \& Diamond, M. S. Memory B cells, but not long-lived plasma cells, possess antigen specificities for viral escape mutants. J. Exp. Med. 208, 2599-2606 (2011).

This study finds that MBCs, but not plasma cells, can recognize previously unencountered stains of the West Nile virus, suggesting that MBCs might be an important component of heterosubtypic immunity

10. Andrade, P. et al. Impact of pre-existing dengue immunity on human antibody and memory B cell responses to Zika. Nat. Commun. 10, 938 (2019).

11. McCarthy, K. R. et al. Memory B cells that cross-react with group 1 and group 2 influenza A viruses are abundant in adult human repertoires. Immunity 48, 174-183 e9 (2018)

12. Leach, S. et al. Requirement for memory B-cell activation in protection from heterologous influenza virus reinfection. Int. Immunol. 31, 771-779 (2019).

13. Benson, M. J. et al. Distinction of the memory $\mathrm{B}$ cell response to cognate antigen versus bystander inflammatory signals. J. Exp. Med. 206, 2013-2025 (2009).

14. Krishnamurty, A. T. et al. Somatically hypermutated plasmodium-specific $\operatorname{lgM}^{+}$memory B cells are rapid, plastic, early responders upon malaria rechallenge. Immunity 45, 402-414 (2016).

15. Zabel, F. et al. Viral particles drive rapid differentiation of memory B cells into secondary plasma cells producing increased levels of antibodies. J. Immunol. 192, 5499-5508 (2014).

16. Mesin, L. et al. Restricted clonality and limited germinal center reentry characterize memory $B$ cell reactivation by boosting. Cell 180, 92-106.e11 (2020).

This study finds that most MBC clones fail to re-enter the $G C$ during recall responses. Rather, recall GCs tend to consist largely of $B$ cells with no GC experience.

17. Zhang, Y. et al. Germinal center B cells govern their own fate via antibody feedback. J. Exp. Med. 210 457-464 (2013).

18. Toyama, H. et al. Memory B cells without somatic hypermutation are generated from $\mathrm{Bcl}$ 6-deficient B cells. Immunity 17, 329-339 (2002).

19. Obukhanych, T. V. \& Nussenzweig, M. C. T-independent type II immune responses generate memory B cells. J. Exp. Med. 203, 305-310 (2006).

20. Pritchard, G. H. et al. The development of optimally responsive plasmodium-specific $\mathrm{CD} 73+{ }^{+} \mathrm{CD} 80^{+}+\mathrm{IgM}^{+}$ memory $B$ cells requires intrinsic BCL6 expression but not CD4 $\mathrm{T}_{\mathrm{FH}}$ cells. Preprint at bioRxiv https://doi.org/ 10.1101/564351 (2019).

21. Kenderes, K. J. et al. T-Bet IgM memory cells generate multi-lineage effector B cells. Cell Rep. 24, 824-837. e3 (2018).

22. Fukuda, T. et al. Disruption of the Bcl6 gene results in an impaired germinal center formation. J. Exp. Med. 186, 439-448 (1997). 
23. Huang, C. et al. The BCL6 RD2 domain governs commitment of activated B cells to form germinal centers. Cell Rep. 8, 1497-1508 (2014)

24. Willis, S. N. et al. Bcl6 protein expression shapes pregerminal center $\mathrm{B}$ cell dynamics and follicular helper T cell heterogeneity. J. Immunol. 192, 3200-3206 (2011)

25. Basso, K. et al. Integrated biochemical and computational approach identifies BCL6 direct target genes controlling multiple pathways in normal germinal center B cells. Blood 115, 975-984 (2010).

26. Saito, M. et al. BCL6 suppression of BCL2 via Miz 1 and its disruption in diffuse large B cell lymphoma. Proc. Natl Acad. Sci. USA 106, 11294-11299 (2009).

27. Li, Z. et al. BCL-6 negatively regulates expression of the NF-кB1 p105/p50 subunit. J. Immunol. 174, 205-214 (2004).

28. Linterman, M. A. et al. IL-21 acts directly on B cells to regulate $\mathrm{Bcl}-6$ expression and germinal center responses. J. Exp. Med. 207, 353-363 (2010)

29. Chevrier, S., Kratina, T., Emslie, D., Tarlinton, D. M. \& Corcoran, L. M. IL4 and IL21 cooperate to induce the high Bcl6 protein level required for germinal center formation. Immunol. Cell Biol. https://doi.org/ 10.1038/icb.2017.71 (2017)

30. Zotos, D. et al. IL-21 regulates germinal center B cell differentiation and proliferation through a B cellintrinsic mechanism. J. Exp. Med. 207, 365-378 (2010)

31. Avery, D. T. et al. B cell-intrinsic signaling through IL-21 receptor and STAT3 is required for establishing long-lived antibody responses in humans. J. Exp. Med. 207, 155-171 (2010).

32. Ochiai, K. et al. Transcriptional regulation of germinal center $\mathrm{B}$ and plasma cell fates by dynamical control of IRF4. Immunity 38, 918-929 (2013).

33. Klein, U. et al. Transcription factor IRF4 controls plasma cell differentiation and class-switch recombination. Nat. Immunol. 7, 773-782 (2006).

34. Willis, S. N. et al. Transcription factor IRF4 regulates germinal center cell formation through a B cellintrinsic mechanism. J. Immunol. 192, 3200-3206 (2014).

35. Sciammas, R. et al. An incoherent regulatory network architecture that orchestrates B cell diversification in response to antigen signaling. Mol. Syst. Biol. 7, 495 (2011).

36. Saito, M. et al. A signaling pathway mediating downregulation of BCL6 in germinal center B cells is blocked by BCL6 gene alterations in B cell lymphoma. Cancer Cell 12, 280-292 (2007)

37. Sciammas, R. et al. Graded expression of interferon regulatory factor-4 coordinates isotype switching with plasma cell differentiation. Immunity 25, 225-236 (2006).

38. Lee, C. H. et al. Regulation of the germinal center gene program by interferon (IFN) regulatory factor 8/IFN consensus sequence-binding protein. J. Exp. Med. 203, 63-72 (2006)

39. Zhou, J. X. et al. IFN regulatory factor 8 regulates MDM2 in germinal center B cells. J. Immunol. 183 3188-3194 (2009).

40. Yoon, J. et al. Interferon regulatory factor 8 (IRF8) interacts with the B cell lymphoma 6 (BCL6) corepressor BCOR. J. Biol. Chem. 289 , 34250-34257 (2014).

41. Carotta, S. et al. The transcription factors IRF8 and PU.1 negatively regulate plasma cell differentiation. J. Exp. Med. 211, 2169-2181 (2014).

42. Wang, H. et al. Transcription factors IRF8 and PU.1 are required for follicular $\mathrm{B}$ cell development and BCL6-driven germinal center responses. Proc. Natl Acad. Sci. USA 116, 9511-9520 (2019).

43. Schubart, D. B., Rolink, A., Kosco-Vilbois, M. H. Botteri, F. \& Matthias, P. B-cell-specific coactivator OBF-1/OCA-B/Bob1 required for immune response and germinal centre formation. Nature 383 . 538-542 (1996)

44. Kim, U. et al. The B-cell-specific transcription coactivator OCA-B/OBF-1/Bob-1 is essential for normal production of immunoglobulin isotypes. Nature 383, 542-547 (1996).

45. Corcoran, L. et al. Oct2 and Obf1 as facilitators of $B: T$ cell collaboration during a humoral immune response. Front. Immunol. 5, 108 (2014).

46. Karnowski, A. et al. B and T cells collaborate in antiviral responses via IL-6, IL-21, and transcriptional activator and coactivator, Oct2 and OBF-1. J. Exp. Med. 209, 2049-2064 (2012)

47. Bartholdy, B. et al. The Ets factor Spi-B is a direct critical target of the coactivator OBF-1. Proc. Nat Acad. Sci. USA 103, 11665-11670 (2006).
48. Garrett-Sinha, L. A. et al. PU.1 and Spi-B are required for normal B cell receptor-mediated signal transduction. Immunity 10, 399-408 (1999).

49. Su, G. H. et al. Defective B cell receptor-mediated responses in mice lacking the Ets protein, Spi-B. EMBO J. 16, 7118-7129 (1997)

50. Willis, S. N. et al. Environmental sensing by mature B cells is controlled by the transcription factors PU.1 and SpiB. Nat. Commun. 8, 1426 (2017).

51. Calado, D. P. et al. The cell-cycle regulator c-Myc is essential for the formation and maintenance of germinal centers. Nat. Immunol. 13, 1092-1100 (2012).

52. Dominguez-Sola, D. et al. The proto-oncogene MYC is required for selection in the germinal center and cyclic reentry. Nat. Immunol. 13, 1083-1091 (2012).

53. Tanaka, H. et al. E2F1 and c-Myc potentiate apoptosis through inhibition of NF-kB activity that facilitates MnSOD-mediated ROS elimination. Mol. Cell 9 1017-1029 (2002)

54. Béguelin, W. et al. EZH2 enables germinal centre formation through epigenetic silencing of CDKN 1A and an Rb-E2F1 feedback loop. Nat. Commun. 8, 877 (2017).

55. Brescia, P. et al. MEF2B instructs germinal center development and acts as an oncogene in B cell lymphomagenesis. Cancer Cell 34, 453-465.e9 (2018).

56. Wilker, P. R. et al. Transcription factor Mef2c is required for $\mathrm{B}$ cell proliferation and survival after antigen receptor stimulation. Nat. Immunol. 9, 603-612 (2008)

57. Khiem, D., Cyster, J. G., Schwarz, J. J. \& Black, B. L. A p38 MAPK-MEF2C pathway regulates B-cell proliferation. Proc. Natl Acad. Sci. USA 105 17067-17072 (2008).

58. Ying, C. Y. et al. MEF2B mutations lead to deregulated expression of the oncogene BCL6 in diffuse large B cell lymphoma. Nat. Immunol. 14, 1084-1092 (2013).

59. Kwon, K. et al. Instructive role of the transcription factor E2A in early B lymphopoiesis and germinal center B cell development. Immunity 28, 751-762 (2008).

60. Wöhner, M. et al. Molecular functions of the transcription factors E2A and E2-2 in controlling germinal center $\mathrm{B}$ cell and plasma cell development. J. Exp. Med. 213, 1201-1221 (2016).

61. Gloury, R. et al. Dynamic changes in Id3 and E-protein activity orchestrate germinal center and plasma cell development. J. Exp. Med. 213, 1095-1111 (2016).

62. Chen, S. et al. Id3 orchestrates germinal center B cell development. Mol. Cell. Biol. 36, 2543-2552 (2016)

63. Green, M. R. et al. Signatures of murine B-cell development implicate Yy1 as a regulator of the germinal center-specific program. Proc. Natl Acad. Sci. USA 108, 2873-2878 (2011).

64. Vilagos, B. et al. Essential role of EBF1 in the generation and function of distinct mature $B$ cell types. J. Exp. Med. 209, 775-792 (2012).

65. Gyory, I. et al. Transcription factor Ebf1 regulates differentiation stage-specific signaling, proliferation, and survival of B cells. Genes. Dev. 26, 668-682 (2012).

66. Huang, C., Geng, H., Boss, I., Wang, L. \& Melnick, A Cooperative transcriptional repression by BCL6 and $\mathrm{BACH} 2$ in germinal center B-cell differentiation. Blood 123, 1012-1020 (2014).

67. Shaffer, A. L. et al. BCL-6 represses genes that function in lymphocyte differentiation, inflammation, and cell cycle control. Immunity 13, 199-212 (2000).

68. Reljic, R., Wagner, S. D., Peakman, L. J. \& Fearon, D. T. Suppression of signal transducer and activator of transcription 3-dependent B lymphocyte terminal differentiation by BCL-6. J. Exp. Med. 192, 1841-1848 (2000).

69. Polo, J. M., Ci, W., Licht, J. D. \& Melnick, A. Reversible disruption of BCL6 repression complexes by CD40 signaling in normal and malignant B cells. Blood 112 644-651 (2008)

70. Niu, H., Ye, B. H. \& Dalla-Favera, R. Antigen receptor signaling induces MAP kinase-mediated phosphorylation and degradation of the BCL-6 transcription factor. Genes. Dev 12, 1953-1961 (1998).

71. Zhang, T.-T. et al. Germinal center B cell development has distinctly regulated stages completed by disengagement from T cell help. eLife 6, e19552 (2017).

72. Ise, W. et al. T follicular helper cell-germinal center $B$ cell interaction strength regulates entry into plasma cell or recycling germinal center cell fate. Immunity 48, 702-715.e4 (2018).
73. Weinstein, J. S. et al. $\mathrm{T}_{F H}$ cells progressively differentiate to regulate the germinal center response Nat Immunol. 17, 1197-1205 (2016)

74. Li, X. et al. Cbl ubiquitin ligases control B cell exit from the germinal-center reaction. Immunity 48, 530-541. e6 (2018)

75. Davidzohn, N. et al. Syk degradation restrains plasma cell formation and promotes zonal transitions in germinal centers. J. Exp. Med. 217, 26648 (2019)

76. Sohn, H. W., Gu, H. \& Pierce, S. K. Cbl-b negatively regulates $\mathrm{B}$ cell antigen receptor signaling in mature B cells through ubiquitination of the tyrosine kinase Syk. J. Exp. Med. 197, 1511-1524 (2003).

77. Ly, A. et al. Transcription factor T-bet in B cells modulates germinal center polarization and antibody affinity maturation in response to malaria. Cell Rep. 29, 2257-2269.e6 (2019).

78. Guthmiller, J. J., Graham, A C. Zander, R. A. Pope, R. L. \& Butler, N. S. Cutting edge: IL-10 is essential for the generation of germinal center $B$ cell responses and anti-plasmodium humoral immunity. J. Immunol. 198, 617-622 (2017).

79. Rubtsova, K. et al. B cells expressing the transcription factor T-bet drive lupus-like autoimmunity. J. Clin. Invest. 127, 1392-1404 (2017).

80. Jackson, S. W. et al. B cell IFN- $\gamma$ receptor signaling promotes autoimmune germinal centers via cell-intrinsic induction of BCL-6. J. Exp. Med. 213, 733-750 (2016)

81. Sander, S. et al. PI3 kinase and FOXO1 transcription factor activity differentially control $B$ cells in the germinal center light and dark zones. Immunity $\mathbf{4 3}$ 1075-1086 (2015).

82. Dominguez-Sola, D. et al. The FOXO1 transcription factor instructs the germinal center dark zone program. Immunity 43, 1064-1074 (2015).

83. Inoue, T. et al. The transcription factor Foxo 1 controls germinal center $\mathrm{B}$ cell proliferation in response to T cell help. J. Exp. Med. 214, 1181-1198 (2017)

84. Bannard, O. et al. Germinal center centroblasts transition to a centrocyte phenotype according to a timed program and depend on the dark zone for effective selection. Immunity 39, 912-924 (2013).

85. Albright, A. R. et al. TGF $\beta$ signaling in germinal center $B$ cells promotes the transition from light zone to dark zone. J. Exp. Med. 216, 2531-2545 (2019).

86. Laidlaw, B. J. et al. Interleukin-10 from $\mathrm{CD}^{+}$follicular regulatory $\mathrm{T}$ cells promotes the germinal center response. Sci. Immunol. 2, eaan4767 (2017).

87. Koike, T., Harada, K., Horiuchi, S. \& Kitamura, D. The quantity of CD40 signaling determines the differentiation of $B$ cells into functionally distinct memory cell subsets. eLife 8, e44245 (2019).

88. Ise, W. et al. The transcription factor BATF controls the global regulators of class-switch recombination in both B cells and T cells. Nat. Immunol. 12, 536-543 (2011).

89. Finkin, S., Hartweger, H., Oliveira, T. Y, Kara, E. E. $\&$ Nussenzweig, M. C. Protein amounts of the MYC transcription factor determine germinal center $B$ cell division capacity. Immunity 51, 324-336.e5 (2019).

90. Chen, C. et al. Uhrf1 regulates germinal center B cell expansion and affinity maturation to control viral infection. J. Exp. Med. 215, 1437-1448 (2018)

91. Toboso-Navasa, A. et al. Restriction of memory B cell differentiation at the germinal center $B$ cell positive selection stage. J. Exp. Med. 217, 943 (2020).

92. Chou, C. et al. The transcription factor AP4 mediates resolution of chronic viral infection through amplification of germinal center $B$ cell responses. Immunity 45, 570-582 (2016).

93. Luo, W., Weisel, F. \& Shlomchik, M. J. B cell receptor and CD40 signaling are rewired for synergistic induction of the c-Myc transcription factor in germinal center B cells. Immunity 48, 313-326.e5 (2018).

94. Gallagher, E. et al. Kinase MEKK1 is required for CD40-dependent activation of the kinases Jnk and p38, germinal center formation, B cell proliferation and antibody production. Nat. Immunol. 8, 57-63 (2006).

95. Heise, N. et al. Germinal center B cell maintenance and differentiation are controlled by distinct NF- $\mathrm{kB}$ transcription factor subunits. J. Exp. Med. 211 2103-2118 (2014).

96. Grumont, R. J. \& Gerondakis, S. Rel induces interferon regulatory factor 4 (IRF-4) expression in lymphocytes: modulation of interferon-regulated gene expression by rel/nuclear factor-кB. J. Exp. Med. 191, 1281-1292 (2000).

97. Grumont, R. J., Strasser, A. \& Gerondakis, S. B cell growth is controlled by phosphatidylinosotol 3-kinase- 
dependent induction of Rel/NF-kB regulated c-myc transcription. Mol Cell 10, 1283-1294 (2002).

98. De Silva, N. S. et al. Transcription factors of the alternative NF-kB pathway are required for germinal center B-cell development. Proc. Natl Acad. Sci. USA 113, 9063-9068 (2016).

99. Luo, W. et al. The AKT kinase signaling network is rewired by PTEN to control proximal BCR signaling in germinal center B cells. Nat. Immunol. 20, 736-746 (2019)

100. Zhu, Z., Shukla, A., Ramezani-Rad, P., Apgar, J. R. \& Rickert, R. C. The AKT isoforms 1 and 2 drive B cell fate decisions during the germinal center response. Life Sci. Alliance 2, e201900506 (2019).

101. Ersching, J. et al. Germinal center selection and affinity maturation require dynamic regulation of $\mathrm{MTORC}$ kinase Immunity 46, 1045-1058.e6 (2017)

102. Raybuck, A. L. et al. B cell-intrinsic mTORC1 promotes germinal center-defining transcription factor gene expression, somatic hypermutation, and memory B cell generation in humoral immunity. J. Immunol. 200 2627-2639 (2018)

103. Kometani, K. et al. Repression of the transcription factor Bach2 contributes to predisposition of IgG 1 memory B cells toward plasma cell differentiation. Immunity 39, 136-147 (2013).

104. Mayer, C. T. et al. The microanatomic segregation of selection by apoptosis in the germinal center. Science 358, eaao2602 (2017).

105. Stewart, I., Radtke, D., Phillips, B., McGowan, S. J. \& Bannard, O. Germinal center B cells replace their antigen receptors in dark zones and fail light zone entry when immunoglobulin gene mutations are damaging. Immunity 49, 477-489.e7 (2018).

106. Tamahara, T. et al. The mTOR-Bach2 cascade controls cell cycle and class switch recombination during B cell differentiation. Mol. Cell. Biol. 37, e00418-17 (2017).

107. Miura, Y. et al. Bach2 promotes B cell receptor induced proliferation of $B$ lymphocytes and represses cyclin-dependent kinase inhibitors. J. Immunol. 200 2882-2893 (2018)

108. Fischer, S. F. et al. Proapoptotic BH3-only protein Bim is essential for developmentally programmed death of germinal center-derived memory B cells and antibodyforming cells. Blood 110, 3978-3984 (2007).

109. Smith, K. G., Weiss, U., Rajewsky, K., Nossal, G. J. \& Tarlinton, D. M. Bcl-2 increases memory B cell recruitment but does not perturb selection in germinal centers. Immunity 1, 803-813 (1994).

110. Laidlaw, B. J. et al. The Eph-related tyrosine kinase ligand Ephrin-B1 marks germinal center and memory precursor B cells. J. Exp. Med. 214, 639-649 (2017)

111. Wang, Y. et al. Germinal-center development of memory B cells driven by IL-9 from follicular helper T cells. Nat. Immunol. 18, 921-930 (2017).

112. Suan, D. et al. CCR6 defines memory B cell precursors in mouse and human germinal centers, revealing lightzone location and predominant low antigen affinity. Immunity 47, 1142-1153.e4 (2017). Laidlaw et al. (J. Exp. Med., 2017), Wang et al (2017) and Suan et al. identify and characterize $\mathrm{MBC}$ precursors residing in the light zone of the GC in mice and humans.

113. Kuo, T. C. et al. Repression of BCL-6 is required for the formation of human memory B cells in vitro. J. Exp. Med. 204, 819-830 (2007)

114. Hatzi, K. et al. A hybrid mechanism of action for BCL6 in $B$ cells defined by formation of functionally distinct complexes at enhancers and promoters. Cell Rep. 4 578-588 (2013)

115. Bhattacharya, D. et al. Transcriptional profiling of antigen-dependent murine $\mathrm{B}$ cell differentiation and memory formation. J. Immunol. 179, 6808-6819 (2007).

116. Xing, S. et al. Tle corepressors are differentially partitioned to instruct CD8 ${ }^{+} \mathrm{T}$ cell lineage choice and identity. J. Exp. Med. 215, 2211-2226 (2018).

117. Jangal, M. et al. The transcriptional co-repressor TLE3 suppresses basal signaling on a subset of estrogen receptor a target genes. Nucleic Acids Res. 42, 11339-11348 (2014)

118. Takatsuka, S. et al. IL-9 receptor signaling in memory B cells regulates humoral recall responses. Nat. Immunol. 19, 1025-1034 (2018).

119. Jash, A. et al. ZBTB32 restricts the duration of memory B cell recall responses. J. Immunol. 197 1159-1168 (2016).
120. Bernasconi, N. L., Onai, N. \& Lanzavecchia, A. A role for Toll-like receptors in acquired immunity: up-regulation of TLR9 by BCR triggering in naive $B$ cells and constitutive expression in memory B cells. Blood 101, 4500-4504 (2003).

121. Onodera, T. Whole-virion influenza vaccine recalls an early burst of high-affinity memory B cell response through TLR signaling. J. Immunol. 196, 4172-4184 (2016).

122. Lutz, J. et al. Reactivation of IgG-switched memory B cells by BCR-intrinsic signal amplification promotes IgG antibody production. Nat. Commun. 6, 8575 (2015).

123. Thomas, M. J., Klein, U., Lygeros, J. \& Rodríguez Martínez, M. A probabilistic model of the germinal center reaction. Front. Immunol. 10, 689 (2019).

124. Wang, P., Shih, C.-M., Qi, H. \& Lan, Y.-H. A stochastic model of the germinal center integrating local antigen competition, individualistic T-B interactions, and B cell receptor signaling. J. Immunol. 197, 1169-1182 (2016).

125. Vikstrom, I. et al. Mcl-1 is essential for germinal center formation and B cell memory. Science 330 1095-1099 (2010).

126. Barnett, B. E. et al. Asymmetric B cell division in the germinal center reaction. Science 335, 342-344 (2012).

127. Lin, W.-H. W. et al. Asymmetric PI3K signaling driving developmental and regenerative cell fate bifurcation. Cell Rep. https://doi.org/10.1016/j.celrep.2015. 10.072 (2015)

128. Duffy, K. R. et al. Activation-induced B cell fates are selected by intracellular stochastic competition. Science 335, 338-341 (2012).

129. Taylor, J. J., Pape, K. A., Steach, H. R. \& Jenkins, M. K. Humoral immunity. Apoptosis and antigen affinity limit effector cell differentiation of a single naïve $B$ cell. Science 347, 784-787 (2015).

130. Tas, J. M. J. et al. Visualizing antibody affinity maturation in germinal centers. Science 351 1048-1054 (2016)

131. Allie, S. R. et al. The establishment of resident memory $B$ cells in the lung requires local antigen encounter. Nat. Immunol. 20, 97-108 (2019). This study finds that MBCs develop in the lungs after influenza infection and rapidly differentiate into local antibody-secreting cells upon challenge infection.

132. Adachi, Y. et al. Distinct germinal center selection at local sites shapes memory B cell response to vira escape. J. Exp. Med. 212, 1709-1723 (2015). This study finds that MBCs in the lungs have an elevated fraction of cross-reactive clones relative to cells in the secondary lymphoid organs after influenza virus infection.

133. Sungalee, S. et al. Germinal center reentries of BCL2overexpressing B cells drive follicular lymphoma progression. J. Clin. Invest. 124, 5337-535 (2014).

134. Schmitz, R. et al. Genetics and pathogenesis of diffuse large B-cell lymphoma. N. Engl. J. Med. 378, 1396-1407 (2018)

135. Chapuy, B. et al. Molecular subtypes of diffuse large B cell lymphoma are associated with distinct pathogenic mechanisms and outcomes. Nat. Med. 24, 679-690 (2018)

136. Venturutti, L. et al. TBL1XR1 mutations drive extranodal lymphoma by inducing a pro-tumorigenic memory fate. Cell https://doi.org/10.1016/j.cell. 2020.05.049 (2020)

This paper reports that a subset of B cell lymphoma is driven by mutations that bias cells to develop into immature MBCs.

137. He, J.-S. et al. IgG1 memory B cells keep the memory of IgE responses. Nat. Commun. 8, 641 (2017).

138. Asrat, S et al. Chronic allergen exposure drives accumulation of long-lived IgE plasma cells in the bone marrow, giving rise to serological memory. Sci. Immunol. 5, eaav8402 (2020).

139. Gowthaman, U. et al. Identification of a T follicular helper cell subset that drives anaphylactic IgE. Science 365, eaaw6433 (2019).

140. Sayegh, C. E., Quong, M. W., Agata, Y. \& Murre, C. E-proteins directly regulate expression of activation induced deaminase in mature B cells. Nat. Immunol. 4, 586-593 (2003).
141. Gonda, H. et al. The balance between Pax 5 and Id2 activities is the key to AID gene expression. J. Exp. Med. 198, 1427-1437 (2003)

142. Tomayko, M. M., Steinel, N. C., Anderson, S. M. \& Shlomchik, M. J. Cutting edge: hierarchy of maturity of murine memory B cell subsets. J. Immunol. 185, 7146-7150 (2010).

143. Onodera, T. et al. Memory B cells in the lung participate in protective humoral immune responses to pulmonary influenza virus reinfection. Proc. Natl Acad. Sci. USA 109, 2485-2490 (2012).

144. Lindner, C. et al. Diversification of memory B cells drives the continuous adaptation of secretory antibodies to gut microbiota. Nat. Immunol. 16, 880-888 (2015).

145. Bar-Or, A. et al. Immunological memory: contribution of memory $B$ cells expressing costimulatory molecules in the resting state. J. Immunol. 167, 5669-5677 (2001).

146. Weisel, F. \& Shlomchik, M. Memory B cells of mice and humans. Annu. Rev. Immunol. 35, 255-284 (2017).

147. Seifert, M. et al. Functional capacities of human IgM memory B cells in early inflammatory responses and secondary germinal center reactions. Proc. Natl Acad. Sci. USA 112, E546-E555 (2015).

148. Siewe, B. Nipper A J., Sohn, H., Stapleton, J. T. $\&$ Landay, A. FCRL4 expression identifies a proinflammatory B cell subset in viremic HIV-infected subjects. Front. Immunol. 8, 117 (2017).

149. Naradikian, M. S. et al. Cutting edge: IL-4, IL-21, and IFN- $\gamma$ interact to govern T-bet and CD11c expression in TLR-activated B cells. J. Immunol. 197, 1023-1028 (2016).

150. Yates, J. L., Racine, R., McBride, K. M. \& Winslow, G. M. T Cell-Dependent IgM Memory B Cells generated during bacterial infection are required for IgG responses to antigen challenge. J. Immunol. 191, 1240-1249 (2013).

151. Jenks, S. A. et al. Distinct effector B cells induced by unregulated Toll-like receptor 7 contribute to pathogenic responses in systemic lupus erythematosus. Immunity 49, 725-739.e6 (2018).

152. Du, S. W. et al. Functional characterization of CD $11 \mathrm{C}^{+}$ age-associated B cells as memory B cells. J. Immunol. 203, 2817-2826 (2019)

153. Kim, C. C., Baccarella, A. M., Bayat, A., Pepper, M. \& Fontana, M. F. FCRL5+ memory B cells exhibit robust recall responses. Cell Rep. 27, 1446-1460.e4 (2019).

154. Peng, S. L., Szabo, S. J. \& Glimcher, L. H. T-bet regulates IgG class switching and pathogenic autoantibody production. Proc. Natl Acad. Sci. USA 99, 5545-5550 (2002).

155. Johnson, J. L. et al. The transcription factor T-bet resolves memory B cell subsets with distinct tissue distributions and antibody specificities in mice and humans. Immunity 52, 841-855.e6 (2020).

156. Piovesan, D. et al. c-Myb regulates the T-betdependent differentiation program in B cells to coordinate antibody responses. Cell Rep. 19 , 461-470 (2017)

157. Stone, S. L. et al. T-bet transcription factor promotes antibody-secreting cell differentiation by limiting the inflammatory effects of IFN- $\gamma$ on B cells. Immunity $\mathbf{5 0}$ 1172-1187.e7 (2019)

158. Rubtsova, K., Rubtsov, A. V., van Dyk, L. F, Kappler, J. W. \& Marrack, P. T-box transcription factor T-bet, a key player in a unique type of B-cell activation essential for effective viral clearance. Proc. Natl Acad. Sci. USA 110, E3216-E3224 (2013).

Author contributions

The authors contributed equally to all aspects of the article

\section{Competing interests}

The authors declare no competing interests.

\section{Peer review information}

Nature Reviews Immunology thank the anonymous reviewers for their contribution to the peer review of this work.

\section{Publisher's note}

Springer Nature remains neutral with regard to jurisdictional claims in published maps and institutional affiliations.

C) Springer Nature Limited 2020 